\title{
DIALECTOLOGÍA Y TOPONIMIA EN LA OBRA DE FLOR GARITA HERNÁNDEZ
}

\section{Dialectology and toponimy in the work of Flor Garita Hernández.}

\author{
Alberto Barahona Novoa*
}

\section{RESUMEN}

Este artículo reseña los estudios sobre la toponimia costarricense publicados por Flor Garita Hernández. Se presentan las tendencias en los procedimientos de nominación de los accidentes geográficos, pueblos, montes, ríos, costas y calles en Costa Rica. Se destaca la importancia del sistema clasificatorio ideado por Hernández para sistematizar los estudios toponímicos. Por otra parte, se resaltan los aportes de sus investigaciones para el conocimiento de la variedad dialectal hispánica de Costa Rica.

Palabras clave: Toponimia, geografía lingüística, dialectología, español de Costa Rica, Garita Hernández.

\begin{abstract}
This article reviews the studies on Costa Rican toponymy published by Flor Garita Hernández. The trends in the nomination procedures for geographic features, towns, mountains, rivers, coasts and streets in Costa Rica are presented. The importance of the classificatory system devised by Hernández to systematize the toponymic studies is highlighted. On the other hand, the contributions of his investigations for the knowledge of the Hispanic dialectal variety of Costa Rica are highlighted.

Key Words: Toponymy, linguistic geography, dialectology, Costa Rican Spanish, Garita Hernández.
\end{abstract}

\section{Toponimia, geolingüística y dialectología}

Es harto conocida la relación decimonónica entre la geolingüística o geografía lingüística y la dialectología. Las relaciones entre ambas subdisciplinas fueron múltiples y complementarias. Los hallazgos de la primera de ellas constituyen amplios bancos de datos de los diversos niveles lingüísticos por zonas geográficas, configurando así perfiles dialectales obtenidos de primera mano, cuya existencia propició la reflexión teórica que llevó a establecer la teoría de las áreas lingüísticas. Además, demostró la conveniencia de desarrollar una metodología específica, pues uniformaba el tratamiento de los datos y su posterior comparación, así como el afinamiento de conclusiones teóricas. Por último, se propiciaron los estudios onomasiológicos de puntos geográficos concretos.

\footnotetext{
* Universidad de Costa Rica. Profesor catedrático del Departamento de la Escuela de Filología, Lingüística y Literatura. Costa Rica. Correo electrónico: jose.barahona@ucr.ac.cr
}

Recepción: 22/10/2017. Aprobación: 28/01/2019. 
Sin embargo, el vínculo más prominente y, a la vez, más obvio es el acercamiento de los estudios geográficos y lingüísticos. En esta confluencia de saberes, los estudios toponímicos son un componente esperable y deseable, pues la onomástica de los accidentes geográficos y asentamientos humanos se convierte en guía y primera herramienta de trabajo para organizar los estudios de la variación lingüística 1 horizontal y, en consecuencia, de las identidades lingüísticas de las comunidades de habla, que no es otra cosa que dialectología pura. A propósito de esta tarea colaborativa, Pilar García Mouton advierte, en referencia a los trabajos de Jules Gilliéron, lo siguiente:

Ahora bien, con ser extraordinario el trabajo del ALF,2 lo que más revolucionó el panorama lingüístico europeo fueron los trabajos posteriores de Gilliéron, en los que estudiaba de una forma desconocida hasta entonces, más que los dialectos en sí, la vida de la lengua a partir de esos mapas, reconstruyendo las situaciones anteriores a la que aparecía cartografiada (García Mouton, 1996, p. 65).

Es imperioso destacar también que los estudios geolingüísticos revelaron que el estudio de los materiales recolectados en el trabajo de campo y luego cartografiados no se agota en la descripción de su evolución fonética, sino que deben atenderse todos los factores que intervienen en su adopción como elemento normal de uso en una comunidad; en otras palabras, ha de tenerse en cuenta que cada palabra tiene su propia historia.

En el ámbito hispanoamericano, al revisar la historia de su lexicografía, surgen dos obras fundacionales que mucho antes al siglo XIX habían propuesto que el conocimiento de los dialectos americanos está ineludiblemente asociado a la consideración de la toponimia.3

1 En la metodología de la geografía lingüística, se impone la creación de la red de encuesta, es decir, el conjunto de localidades en las que se obtiene la información por medio de encuestas.

2 Siglas de Atlas linguistique de la France.

3 Esta información fue obtenida en la obra de Miguel Ángel Quesada Pacheco, titulada El español de América (2002), en su capítulo I "El español de América: historia de un concepto", cuando se refiere a la primera etapa de evolución de dicho concepto. 
Entre 1750 y 1777, el panameño Manuel José de Ayala redacta el Diccionario de Voces Americanas que, para muchos, es la obra pionera de la lexicografía americana. Este diccionario recopila cerca de 2800 voces, de las cuales muchos son topónimos. Destáquese aquí la importancia que adquiere para la diferenciación del español americano el aporte de la toponimia.

Pocos años después (de 1786 a 1789), Antonio de Alcedo publica el Diccionario histórico geográfico de la Indias Occidentales. Tiene pocas diferencias con el diccionario de Ayala; su principal semejanza se debe a la masiva presencia de topónimos. En este caso, es evidente la importancia que se les otorga a los aspectos histórico-geográficos para comprender el español americano.

En el acervo moderno de la investigación lingüística costarricense, la obra de Flor Garita Hernández debe ser considerada pionera por el grado de exhaustividad y sistematización de los datos heurísticos que surgen de la intersección de la geografía y la lingüística. Sus publicaciones sin ser estrictamente planteadas como obras de geografía lingüística, pero sí derivadas del análisis de datos de la onomástica de accidentes geográficos y asentamientos humanos (topónimos, hidrónimos, ecónimos, litónimos y orónimos), desarrollan, a pie juntillas, las facetas que han enriquecido la dialectología desde el análisis de los atlas lingüísticos. Sus estudios de la onomástica geográfica costarricense han permitido, sin duda alguna, un óptimo conocimiento de los dialectos hispánicos de Costa Rica, aún mejor de los usos lingüísticos de las comunidades de habla nacionales: en definitiva, de nosotros mismos como seres de palabras. 


\section{Toponimia de las provincias de Costa Rica: ingente banco de datos}

La colección de libros de topónimos de Costa Rica constituye, por sí sola, un inmenso banco de datos, propicio como material de estudio para investigar la variación dialectal en el país. Ofrece varias ventajas; pues, a parte de la recolección de lexemas, estos se encuentran tratados mediante un esquema clasificatorio semántico propuesto por la miasma autora, en la primera de las publicaciones.

Esta serie de publicaciones sistematizan la toponimia de cinco de las provincias costarricenses, a saber: Alajuela, Cartago, Guanacaste, Heredia y Puntarenas. Las provincias de San José y Limón fueron tratadas por otros investigadores como trabajos finales de graduación, bajo la guía de la profesora Garita Hernández.

La autora, por sí misma, ha probado la fecundidad de la recolecta de estas voces y su inigualable capacidad de convertirse en base para investigaciones de muy diversa índole, ya que también ha publicado artículos especializados, cuyo corpus emerge de la recolección de términos recogidos en estas publicaciones.

Con la investigación lingüística de Garita Hernández, la toponimia costarricense deja su lecho cartográfico descriptivo para convertirse en materia viva de análisis para la comprensión de las comunidades que han integrado su entorno de residencia a su particular cosmovisión, mediante la apropiación de un sistema lingüístico y adaptación de sus recursos a la realidad inmediata. Además, la sistematización aplicada a los tradicionales listados de nombres geográficos -por lo general, ordenados simplemente por criterio ortográfico- permite comprender ahora el criterio de 
aprehensión de la realidad, conocer las categorías de palabras más frecuentemente utilizadas, así como los recursos linguiísticos para la formación de nuevos vocablos.

\subsection{Toponimia de la provincia de Cartago, 1995}

Esta publicación es la primera de la serie de obras dedicadas a la toponimia costarricense. Con ella se echan las bases que guiarán este aporte al conocimiento de la onomástica costarricense.

Antes de comentar los resultados, es menester referirse al modelo de clasificación propuesto en esta obra, el cual es pionero en este campo. Los estudios previos sobre topónimos se caracterizaban por su naturaleza especialmente descriptiva. Con esta propuesta, se alcanza la sistematización teórica, que pretende rebasar las limitaciones del historicismo y alcanzar una aproximación a la conciencia del hablante; es decir, establecer un modelo ontogenético que explique cómo surgen los nombres. Con él, se logró, dentro de los estudios lingüísticos costarricenses, realizar generalizaciones sobre la toponimia, así como comparaciones entre los resultados obtenidos. Además, esta propuesta teórica permite apreciar las preferencias por determinadas estructuras gramaticales; así como los componentes culturales involucrados. En el caso de América, resulta sintomático la abundancia de hagiónimos de la fe católica.

Este modelo, posteriormente aplicado al análisis de la onomástica de otras provincias, rindió los resultados esperados, al punto de que la autora en fecha posterior concluye: "el aporte fundamental de este modelo consiste en analizar los hábitos lingüísticos de la gente que se enfrenta con la necesidad de producir topónimos” (Garita Hernández y Bedoya Benítez, 2010, p. 253).

En cuanto a la toponimia cartaginesa, se logran establecer constantes en la selectividad de las formas lingüísticas, como las que siguen: preferencia de uso de sustantivos simples singulares, así como el de frases especificativas formadas por sustantivo y adjetivo. Por su parte, en cuanto a la 
relación entre la lengua y la cultura se destacan los siguientes hechos: baja presencia de aportes de la cultura indígena, uso profuso de hagiónimos pertenecientes a la fe católica profesada en la cultura hispánica tradicional (cerro Noche Buena). Para esta provincia, se debe mencionar en especial la referencia a la Virgen de los Ángeles. También sobresale la preeminencia de la familia y la propiedad privada como rasgos culturales identitarios (finca Cristina).4 Por último, se destacan las relaciones entre el hombre y la naturaleza (cerro Mirador).

\subsection{Toponimia de la provincia de Heredia, 1997}

Los resultados de esta investigación siguen muy de cerca a los del estudio de la toponimia de Cartago. En resumen, nos explica Garita Hernández:

El comportamiento del hablante en la selectividad de la forma lingüística es el mismo: preferencia por los sustantivos simples y las frases; por las formas simples a las prefijadas o sufijadas, y, a diferencia de Cartago, en Heredia predominan las construcciones con sufijos diminutivos (Garita Hernandez, 1997, p. 87).

Del examen de la relación lengua-cultura, en cuanto al componente prehispánico, se encuentran muy pocas evidencias. Incluso en algunos vocablos de presumida herencia huetar, su significado es totalmente incierto.

Dentro del componente hispánico, los antropónimos -en especial los hagiónimos- ocupan el primer lugar de frecuencia. El nombre más recurrente es San Rafael. Cuando se escogen nombres de pila, los nombres femeninos son los preferidos. Se evidencia así que estas dos categorías -hagiónimos y nombres de mujer-son las constantes dentro del aporte de la herencia hispánica.

En el caso de esta provincia, se destacan dos hechos curiosos con respecto a las otras provincias: dos poblados reciben nombres de origen peculiar: Chiripa es nominado así por una pulpería; Conservatorio Castella, por una institución educativa ubicada en las cercanías del lugar.

4 La autora encuentra en la tendencia a nominar con nombres de miembros de la familia o de parientes, que la 
Como punto final, se incluyen las anécdotas que constituyen un banco amplísimo de información etnolingüística.

\subsection{Toponimia de la provincia de Guanacaste, 2000}

En este libro, se presenta la toponimia de una zona dialectal históricamente distinguida de la vallecentraleña, también considerada por varios dialectólogos como una realidad perteneciente a una macro zona o continuo dialectal compartido con dialectos costeros del pacífico nicaragüense y panameño.

Esta diferenciación, que para algunos podría ser antagonismo, crea una expectativa por los posibles resultados divergentes con respecto al centro del país. La profesora Garita Hernández (2000), empero, luego de una exhaustiva labor de análisis lingüístico ontogenético, afirma: "El comportamiento del hablante en la selectividad de la forma lingüística que afecta los tipos de términos y su frecuencia coincide con lo investigado para las provincias de Cartago y Heredia” (p. 176).

En dicho análisis, se determina que los sustantivos simples singulares son las formas preferidas para la nominación (quebrada Caleta). Esta es una tendencia que se mantiene en todas las provincias. Constituye así uno de los logros heurísticos de aplicar sistemáticamente una metodología uniforme al tratamiento de la onomástica. También se repite la predilección de uso de las frases especificativas formadas por artículo y sustantivo, cuando se echa mano a estructuras complejas (finca La Ceibita).

familia constituye un valor primordial en la estructura social costarricense. 
En cuanto a la sufijación de género, sobresale la presencia de varios alomorfos para expresar femenino: -a, -esa e -ina (quebrada Maravilla, quebrada Duquesa, río Gallina). Tampoco figuran infijos en el corpus. Con respecto al uso de sufijos abundanciales, el comportamiento es similar a la provincia de Cartago.

El aporte de las lenguas indígenas es reducido, con la particularidad de que la mayoría de voces provenientes de ellas está constituida por nombres de caciques (ciudad de Nicoya), casi todos pertenecientes a la lengua chorotega. Se atestiguan otras voces de origen indígena (playa Bejucos), pero incorporadas al castellano de los conquistadores españoles. Otros aportes ínfimos corresponden a la lengua huetar y a la corobicí (poblado Bagaces). En algunos casos de vocablos de apariencia indígena, queda pendiente el análisis filogenético, pues por ahora no se cuenta con una explicación consistente desde el punto de vista lingüístico, sostiene Garita Hernández.

En las voces procedentes de la tradición hispánica, Garita logra resaltar algunos patrones: 1) preeminencia de nombres de pila, apellidos, apodos e hipocorísticos; 2) alta frecuencia de apellidos, seguidos de nombres de mujer. El concepto de familia como núcleo de la sociedad es un componente compartido con las provincias de Cartago y Heredia (estero Roberto), 5 así como la abundancia de hagiónimos, sobre todo los referidos a santos de la tradición católica.

En Guanacaste hay nombres duplicados de hidrónimos u orónimos en ecónimos, que la autora agrupa en una categoría denominada "castellanos del área"; sin embargo, también hay nombres extranjeros que pueden motivar esta forma de nominalización. De esta forma, se explican nombres como: Arizona, Italia, Alemania y Lombardía.

5 Ver nota anterior. 
Dentro del campo de los nombres que reflejan el impacto de la naturaleza en el hombre, en Guanacaste son mayoría los nombres relacionados con la flora, aspecto que establece una diferencia con Cartago y Heredia.

Los datos aportados por los informantes en relación con la toponimia se agrupan en un último capítulo, el cual es una cantera de información etnolingüística de inapreciable valor para el conocimiento de la identidad lingüística costarricense y, en específico, de la guanacasteca.

\subsection{Toponimia de la provincia de Puntarenas, 2002}

Tan solo dos años después del examen de la onomástica guanacasteca, sale a luz esta investigación elaborada en forma conjunta con Humberto Cordero Romero.

Gracias a este nuevo proyecto y a la aplicación de la metodología creada en el examen de la toponimia cartaginesa, se llega a la siguiente conclusión:

Como en las investigaciones anteriores, el aporte fundamental de este análisis consiste en destacar los hábitos lingüísticos de los hablantes cuando se enfrentan al hecho de crear topónimos. Al respecto se determinó que la gente tiene dos opciones: usar un vocablo que ya existe como nombre propio o tomar elementos comunes del ambiente. De hecho, estos últimos constituyen mayoría (Garita Hernández y Cordero Romero, 2002, p. 252).

En esta investigación, se comprueba nuevamente la existencia de un patrón en la selectividad de las formas lingüísticas, por cuanto no se aprecian diferencias sustanciales con las provincias de Cartago, Heredia y Guanacaste. No obstante, se destaca una diferencia con respecto a ellas, en la provincia de Puntarenas se prefiere nombrar el poblado o la finca con un sustantivo y su respectivo adjetivo calificativo. 
Por la presencia de territorios indígenas en la provincia de Puntarenas, se explica el lugar destacado que ocupa la toponimia indígena, sobre todo de origen cabécar, bribri y boruca. Por su parte, dentro de la tradición hispánica, se distinguen las constantes ya apuntadas: preferencia por los nombres, sean propios o comunes. Cabe destacar que, en este caso, existe equilibrio entre los hagiónimos y otros nombres, e incluso hay muy pocas referencias a la Virgen María o a Dios, lo cual constituye una particularidad de esta zona.

\subsection{Toponimia de la provincia de Alajuela, 2010}

En coautoría con Eduardo Bedoya Benítez, la máster Garita Hernández presenta a la comunidad nacional el estudio correspondiente a la provincia de Alajuela, cuyos resultados se compendiarán a continuación.

En el plano de la morfología, destaca el uso preferente de sustantivos para la denominación de hidrónimos, ecónimos y orónimos, en consonancia con la función de esta categoría de palabras. Luego, pasa a analizar las formas y construcciones más recurrentes, las más escasas y las particularidades encontradas. Destaca el uso del sustantivo simple y la frase especificativa formada por artículo y sustantivo, como las estructuras más frecuentes. Señala el mínimo uso de frases apositivas. Prima, con respecto a otras formas de derivación, la sufijación -sobre todo “abundancial” y “diminutiva”-; por su parte, la infijación es nula.

Dentro de los patrones en la relación lengua-cultura, logra determinar la reducida presencia de nombres indígenas en el corpus correspondiente a esta provincia. En cambio, la influencia hispánica es preponderante; sobre todo las voces procedentes de nombres (fila Delio Herrera) y, entre ellos, los hagiónimos son muy frecuentes como en Heredia, Cartago y Guanacaste. La referencia a San Rafael alcanza el 8,73\% de los nombres de santos utilizados en la toponimia de esta provincia. 
También son abundantes los nombres de pila de mujer (río Elba). En general, se concluye que este rasgo sigue la tradición cultural hispánica.

Por último, en Alajuela como en Guanacaste, funciona el procedimiento llamado "castellano del área" 6 y los nombres "foráneos". Asimismo, se encuentra que el impacto de la naturaleza en el ser humano (poblado Bellavista) es una gran fuente de nombres para la toponimia.

\subsection{Toponimia de la red vial de la Gran Área Metropolitana, 2015}

Esta obra, cuya autoría comparte con el señor Eduardo Bedoya Benítez, no se refiere a la toponimia de una provincia, sino a la red vial de la zona metropolitana; no por ello deja de ser parte de este banco de datos de conocimiento del español costarricense. Se trata de un complemento a la investigación llevada a cabo siguiendo un criterio diferente de organización; además, se amplía el objeto de estudio, pues se incluyen los odónimos. Asimismo, en cuanto a la selectividad de las formas lingüísticas, descuella, en este caso, la aparición del nombre genérico como elemento inicial de las frases. Además, los morfemas abundanciales prevalecen como en investigaciones anteriores. Por último, destacan los autores la inexistencia de nombres para muchas vías.

En cuanto a la participación del componente hispánico, advierten los autores las siguientes particularidades:

Respecto del componente hispánico, como cultura dominante, se invierte la proporción entre nombres propios y nombres comunes; en las investigaciones realizadas por provincias, los nombres comunes son mayoría mientras que en las calles se convierten en minoría. Este hecho se podría justificar por un rasgo característico de las vías que sirven para rendirles homenaje a personajes destacados de Costa Rica o de otros países. Otra razón podría ser que muchas vías de la GAM no tienen nombre, aunque estén enumeradas (Garita Hernández y Bedoya Benítez, 2015, p. 108).

6 La autora explica en qué consiste este procedimiento, de la siguiente manera: "En cuanto a los nombres procedentes de topónimos, se destaca el hábito de duplicar nombres de hidrónimos u orónimos en los ecónimos. Este procedimiento, que se ha denominado 'castellano del área', constituye en esta sección el uso más frecuente" (Garita Hernández, 2010, p. 256) 
Los hagiónimos dejan de ser mayoría y son desplazados por los nombres de pila y apellidos (avenida Eladio Prado Sáenz). Surge, en el campo de los odónimos, una peculiaridad: se registran seudónimos (avenida Carmen Lyra), en baja frecuencia; pero significativos como rasgo cultural. Además, es notoria la ausencia de nombres femeninos, diferencia con la toponimia provincial donde sí son frecuentes como tributo a la madre, a la esposa o a las hijas.

En esta investigación, se eliminó del modelo clasificatorio la casilla correspondiente a "procedentes de topónimos indígenas", ya que no se encontró ningún nombre indígena asignado a una calle. Figuran novedades como el uso de siglas (avenida Unesco) para estos fines.

\subsection{Investigaciones dirigidas sobre la toponimia costarricense}

Además de su labor como investigadora, la magistra Garita Hernández, como parte de su labor docente, dirigió dos trabajos finales de graduación en esta especialidad. Con ellos se logró completar el estudio de todas las provincias nacionales.

En el 2010, la tesiaria Giselle Chang presenta su tesis de maestría en lingüística, titulada: Toponimia de la provincia de Limón. Pocos años después, en el 2013, Yamileth Garbanzo, Marcela Morera, Rosa María Segura y Jessica Zeledón presentan la memoria de graduación para optar al grado de Licenciatura en Filología Española: Toponimia de las urbanizaciones de la Gran Área Metropolitana (GAM) de Costa Rica.

En ambas publicaciones, se logran importantes resultados que completan el conocimiento de la variedad dialectal vernácula. Estas tesis se nutren del modelo ontogenético desarrollado por Garita Hernández, quien con su acertada dirección logra articular la investigación con la docencia universitaria. 


\section{Conocimiento dialectal derivado}

Con la recolección de topónimos en escala nacional, se logró -como ya se evidenció- conformar un copioso banco de datos, el cual se convierte en campo fértil para desarrollar nuevas investigaciones. La acuciosidad de la máster Garita Hernández no le permitía dejar de aprovecharlo; por eso, ella misma se da a la tarea de explotar ese recurso que se materializa en la publicación de varios artículos especializados sobre los topónimos. Estas publicaciones permiten una mejor comprensión del español costarricense. Es inevitable referirse brevemente a cada uno de ellos.

3.1 Clasificación de los topónimos de la provincia de Cartago procedentes de nombres comunes según su clase o función gramatical (Garita Hernández, 1994)

El objetivo de esta publicación es subrayar algunas constantes morfológicas que se cumplen en la formación de topónimos cartagineses. De hecho, en una visión prospectiva, estos hallazgos se confirmarán en los análisis de otras provincias de Costa Rica. Se señala la preferencia por el sustantivo simple singular. Puede aparecer sufijación y prefijación, pero no simultáneamente. Hay un predominio de procesos de sufijación, en especial para expresar abundancia, seguidos por los de tipo apreciativo. Entre los tipos de frases, las más recurrentes son las formadas por sustantivo y adjetivo.

\subsection{Toponimia indígena de la provincia de Guanacaste, Costa Rica (Garita Hernández,} 1998)

En este artículo, la autora determina que la presencia de voces procedentes de lenguas indígenas es más bien exigua. Se conservan solo algunos nombres de caciques, casi todos pertenecientes a la lengua chorotega. No obstante, tomando en cuenta las apreciaciones del Dr. Quesada Pacheco sobre el español de Guanacaste, concluye que:

La escasa contribución chorotega en el enriquecimiento del léxico guanacasteco, se explica por el poco espacio de participación lingüística a que estuvo sometida la cultura chorotega durante la dominación española. / Es también muy reducida la presencia de nombres procedentes de otra lengua autóctona, como lo es la huetar. Pareciera que el término Orosí 
pertenece a la lengua corobicí; sin embargo, esto no ha sido constatado (Garita Hernández, 1998, p. 204).

También se registran voces de lenguas amerindias de otras zonas de América; sin embargo, estas ya estaban integradas al español de los conquistadores y primeros pobladores hispánicos de esta zona costarricense.

La mayoría de las voces tiene como referente nombres de árboles y plantas, ya sea en forma individual o bien abundanciales.

\subsection{Morfemas derivativos y flexivos en la formación toponímica de la provincia de Guanacaste, Costa Rica (Garita Hernández, 2000)}

En este estudio de morfología, se hace un recuento de los formantes más comunes en la constitución de los topónimos de la provincia de Guanacaste. Al respecto, se parte de la presencia tanto de morfemas flexivos como derivativos (loma Abejonal, quebrada Azufrales). En cuanto al género, destaca la irregularidad, pues la mayoría de los topónimos son voces tomadas del ambiente. Sin embargo, los morfemas más comunes son -o y -e para masculino; por su parte, para el femenino se registran -a (quebrada Coyotera), -esa (finca Francesa) e -ina (río Gallina).

En el repertorio de los morfemas derivativos, hay más diversidad, aunque predominan los abundanciales (estero Icacal). Los morfemas con valor despectivo muestran una mayor variedad alomórfica (quebrada Asientillo), seguidos por los diminutivos (poblado Alajuelita), los de acciónefecto y los de cualidad (poblado Angostura). Se utilizan con gran regularidad -er para lugar (quebrada Aserradero) y -ud como intensificador de la cualidad (cerro Brojudo). 


\subsection{La influencia extranjera en la toponimia costarricense (Garita Hernández, 2000)}

En el examen de la toponimia de las provincias nacionales, siempre se encuentra un apartado que recoge la información agrupada bajo la etiqueta nombres "foráneos". De ese arsenal se toma el corpus que analiza y sistematiza la profesora Garita Hernández, en este artículo.

En ninguno de los libros comentados en el apartado 2 se carece de información de este tipo; de hecho, existe una amplia gama de nombres extranjeros tomados de ciudades y países del mundo. En este sentido, cabe destacar que la provincia de Limón es la región con mayor variedad, a criterio de la autora por su conformación multiétnica; en cambio, Heredia presenta la menor influencia de nombres extranjeros.

Las referencias a Europa son numerosas, sin duda alguna por la adscripción de América al hemisferio occidental y los nexos culturales forjados durante la época colonial. En segundo lugar, se ubican las referencias al nombre de Estados Unidos y México, gracias a la influencia que esos dos países norteamericanos ejercen en la cultura costarricense. Solo Guanacaste y Limón cuentan con nombres provenientes del continente asiático. Pocas son las menciones de lugares de Sudamérica o Centroamérica. Hay una gran presencia de nombres bíblicos, quizás debido a la influencia del credo cristiano.

\subsection{Algunos topónimos indígenas de la provincia de Puntarenas, Costa Rica (Garita Hernández, 2001)}

La mayor parte de estos topónimos surge de la interacción del ser humano con su medio. La autora logra establecer las características de esta relación. De los componentes del ambiente abundan elementos de la flora, fauna, objetos o sustancias minerales (el cerro palmera, el río del bejuco de canasta, la sabana con agua e higuerones). Muy pocos nombres se refieren a la actitud que despierta 
el ambiente en el ser humano (p. ej. el cerro que no permite nada); a diferencia de la toponimia no indígena que está llena de estas referencias.

En cuanto a los nombres propios, se menciona lo siguiente:

Un detalle importante y común a estos grupos es el poco uso de nombres propios o comunes que se refieran específicamente a seres humanos. De los nombres bribris registrados solamente uno hace referencia a una deidad que moldea al niño para que sea artesano, agricultor o trabajador con las manos. Entre los borucas encontramos dos nombres que hacen referencia a seres humanos: el cerro Cuasrán y la fila Sankraua, nombres que corresponden, respectivamente, al de un padre y a su hijo, según la leyenda (Garita Hernández, 2001, p. 173).

Este artículo también constituye un aporte al conocimiento de los procedimientos de nominación en las lenguas vernáculas costarricenses, sumándose así a la larga tradición de la investigación lingüística costarricenses sobre estas variedades.

\subsection{Los patrones culturales en la formación de litónimos de Costa Rica (Garita Hernández, 2001)}

Dentro de los estudios toponímicos, esta publicación aborda un nuevo grupo de nombres: los litónimos. Se aplica la metodología ampliamente probada y se alcanzan los siguientes resultados: en primer lugar, la huella prehispánica es muy endeble; por el contrario, de entre las lenguas amerindias actuales ninguna de ellas ha servido para denominar litónimos. Explica Garita Hernández que se debe a que las comunidades indígenas actuales viven en regiones interiores del país. En segundo lugar, del componente hispánico, sobresalen los hagiónimos, aunque no aparecen alusiones a la divinidad y tan solo una a la Virgen María. En cuanto a los nombres propios, se destacan los nombres de mujer. En tercer lugar, se evidencia la baja frecuencia de nombres extranjeros para denominar estos accidentes geográficos. Finalmente, el uso de nombres comunes refleja el impacto de la naturaleza en el hombre. 


\section{Lexicología semántica o la historia de las palabras}

A manera de colofón, las obras publicadas por Garita Hernández ofrecen capítulos que se presentan bajo el título "folclor de los topónimos: explicaciones populares sobre sus orígenes". En estos apartados, se reúne un corpus importantísimo para la investigación de las relaciones lengua y cultura, por lo cual constituyen una veta profunda de conocimiento sobre el español de Costa Rica.

\section{Conclusiones}

En el punto de partida de esta reseña, quedan ya explicadas las relaciones decimonónicas entre la dialectología y la geografía mediatizadas por la llamada geografía lingüística o geolingüística, y cómo los estudios de toponimia de Hernández Garita actualizan y desarrollan los alcances logrados por la confluencia de estos saberes.

Queda clara la importancia del estudio de la toponimia para el conocimiento de las variedades dialectales, tarea atisbada desde muy temprano en la comunidad de intelectuales americanos. Hoy la obra de la señora Flor Garita Hernández permite el conocimiento de nuestra variedad hispánica, de manera fundamentada y actualizada, gracias a su estudio sistemático mediante el esquema clasificatorio propuesto.

Después del examen de los resultados de esta abundante recogida de información a lo largo de todo el país, destaca la homogeneidad en las formas de denominar lugares, ríos, montes, fincas, etc., en todo el país. Quizás esta evidencia sugiere la poca diferenciación dialectal que muestra el español de Costa Rica, por lo menos en el plano léxico asociado a la toponimia. 
Finalmente, es posible deslindar otras conclusiones que nos permiten perfilar aún mejor el español de Costa Rica. Por el estudio de la toponimia, llegamos de nuevo a confirmar que nuestra variedad dialectal posee un componente hispánico fuertemente arraigado, con muy débiles aportes de las lenguas vernáculas costarricenses. Además, la incorporación de voces de lenguas amerindias de otras regiones geográficas confirma la implantación de un español aclimatado a la realidad americana, principalmente por el poblamiento tardío de nuestro territorio.

\section{Referencias bibliográficas}

Chang, G. (2010). Toponimia de la provincia de Limón: tesis para optar al grado de maestría en Lingüística. Universidad de Costa Rica, San José.

Garbanzo, Y., Morera, M., Segura, R., y Zeledón, J. (2013). Toponimia de las urbanizaciones de la Gran Área Metropolitana (GAM) de Costa Rica: memoria de graduación para obtener el grado de licenciatura en Filología Española. Universidad de Costa Rica, San José.

Garita Hernández, F. (2001). Algunos topónimos indígenas de la provincia de Puntarenas, Costa Rica. Revista de Filología y Lingüística, XXVII(2), 157-176.

Garita Hernández, F. (1994). Clasificación de los topónimos de la provincia de Cartago procedentes de nombres comunes segùn su clase o función gramatical. Revista Káñina, XVIII(1), 99-103.

Garita Hernández, F. (2000). La influencia extranjera en la toponimia costarricense. Revista de Filología y Lingüistica, XXVI(2), 139-160.

Garita Hernández, F. (2001). Los patrones culturales en la formación de litónimos de Costa Rica. Kàñina, $X X V(1), 75-89$.

Garita Hernádez, F. (2000). Morfemas derivativos y flexivos en la formación toponímica de la provincia de Guanacaste. Revista de Filología y Lingüística, XXVI(1), 137-155.

Garita Hernández, F. (1995). Toponima de la provincia de Cartago. San José: Editorial de la Dirección de Publicaciones y Editorial de la Universidad de Costa Rica.

Garita Hernández, F. (1998). Toponimia indígena de la provincia de Guanacaste, Costa Rica. Revista de Filología, Lingüística y Literatura, XXIV(2), 177-206.

Garita Hernández, F., y Bedoya Benítez, E. (2010). Toponimia de la provincia de Alajuela. San José: Editorial UCR.

Garita Hernández, F. (2000). Toponimia de la provincia de Guanacaste. San José: Instituto Geográfico Nacional.

Garita Hernandez, F. (1997). Toponimia de la provincia de Heredia. San José: Editorial Instituto Geográfico Nacional. 
Garita Hernández, F., y Cordero Romero, H. (2002). Toponimia de la provincia de Puntarenas. San José: Insituto Geográfico Nacional.

Garita Hernández, F., y Bedoya Benítez, E. (2015). Toponimia de la red vial de la Gran Área Metropolitana. San José: Editorial UCR.

García Mouton, P. (1996). Dialectología y geografía lingüística. En M. Alvar (Ed.), Manual de dialectología hispánica (p. 394). Barcelona, España: Editorial Ariel. 